\title{
Zinc and Dietary Fibre: Competing Nutrients in the Management of Chronic Diseases
}

Samir Samman ${ }^{*}$ Anna Chu and Meika Foster

Department of Human Nutrition, University of Otago, New Zealand

*Corresponding author: Department of Human Nutrition, The University of Otago, PO Box 56 Dunedin 9054, New Zealand, Tel: +64 3479 7945, Fax: +64 3479 7958; E-mail: samir.samman@otago.ac.nz

Received Date: 17 January 2015; Accepted Date: 19 January 2015; Published Date: 26 January 2015

Copyright: ( 2015 Samman S, et al. This is an open-access article distributed under the terms of the Creative Commons Attribution License, which permits unrestricted use, distribution, and reproduction in any medium, provided the original author and source are credited.

\section{Editorial}

Zinc is involved in a number of metabolic processes including the synthesis and action of insulin [1,2]. The primary inhibitor of zinc absorption is phytic acid (PA), which forms poorly soluble complexes with zinc and other minerals in the gastrointestinal tract, resulting in their reduced absorption [3]. The inhibitory effect of PA can be estimated by the molar ratio of PA to zinc in the diet, and WHO has identified 3 grades (low, moderate, high) of zinc bioavailability based on the dietary PA:Zn molar ratio [4]. PA is abundant in unrefined cereals, pulses, and whole grains [5]. Pulses and cereals are major sources of zinc in many populations, and the co-existence of PA and zinc in these staple foods explains much of the association between suboptimal zinc status and plant-based diets. Unrefined pulses, cereals, and whole grains are, in addition, important food sources of dietary fibre. The consumption of a diet that is rich in dietary fibre is recommended as part of healthy eating guidelines and in the dietary management of chronic diseases such as cardiovascular disease and Type 2 diabetes mellitus (DM). Dietary fibre has been shown to decrease plasma cholesterol concentrations and improve glycaemic control [6], and moderate obesity by altering energy expenditure and substrate oxidation [7]. Whilst these outcomes are advantageous in maintaining health and managing disease risk, the presence of PA in fibre-rich foods is likely to lower the bioavailability of zinc in populations that are already at risk of zinc deficiency. The importance of zinc in numerous biological processes and the significant contribution of zinc to the global burden of disease suggest that it warrants special attention when evaluating the nutritional adequacy of therapeutic diets.

\section{Plant-based diets and zinc status}

Plant-based diets contain less saturated fat and cholesterol, and more fibre, folate, and phytochemicals than omnivorous diets; however as alluded to above, zinc is poorly bioavailable when sourced from plant foods. We investigated the effects of plant-based diets on zinc status and showed that dietary zinc intakes and serum zinc concentrations were significantly lower in populations that followed habitual vegetarian diets compared to non-vegetarians. Secondary analyses revealed greater impact of vegetarian diets on the zinc status of females, vegetarians from low- and middle-income countries (LMIC), and vegans $[8,9]$. Although not all vegetarian diets impact zinc status to the same extent, populations that habitually consume vegetarian diets with high PA:Zn molar ratios have a requirement for dietary zinc that is substantially greater than the amount recommended for omnivores.

Methods of food preparation or processing, such as fermentation, soaking and germination, are effective in reducing the impact of PA but these strategies appear to be adopted less commonly in western countries [10]. In addition, an increase in the availability of zincfortified foods is likely to have had a positive impact on the zinc status of some groups, highlighting the need for updated information on zinc bioavailability from a range of diets, particularly in LMIC. Plant breeding practices that decrease the PA content of plant foods [11] and/or the inclusion of phytases in processed foods [12] are options that currently are being explored in the search to maximize zinc bioavailability.

\section{Zinc status in Type 2 diabetes mellitus}

We undertook a study to explore the relationship between PA intake and zinc bioavailability in healthy and DM women [13]. Participants with DM consumed similar amounts of PA as healthy women, and the "breads, cereals, rice, pasta, and noodles" food group on average contributed $55 \%$ of the total daily PA intake. Of this, $21 \%$ of the total PA intake derived from bread and flour products and $21 \%$ from breakfast cereals and oat products, suggesting that a large amount of the daily PA intake is consumed at the morning meal. The PA contribution of vegetables and legumes was significantly different between participant groups; women with DM obtained 26\% of their daily PA from vegetables and legumes compared to $13 \%$ in the healthy group. Nuts and seeds contributed $21 \%$ of the total PA intake in the healthy group and $14 \%$ in those with DM. A positive relationship was observed between PA and dietary fibre in all participants. Based on the WHO classifications, $25 \%, 43 \%$ and $33 \%$ of women consumed diets of high, moderate and low zinc bioavailability, respectively [13]. We concluded that a large percentage of healthy and DM women consume PA in amounts that are likely to decrease zinc bioavailability. Recommendations to consume greater amounts of dietary fibre, much of which is associated with PA, increase the risk of zinc deficiency.

\section{Zinc and glycaemic control}

Zinc has the potential to affect glucose homeostasis via its role in the synthesis and action of insulin, and hence impact the risk of DM [2]. We undertook a meta-analysis of randomised controlled trials to determine the effect of zinc supplementation on glucose and insulin concentrations [14]. We observed a statistically significant reduction in fasting glucose concentrations following zinc supplementation and, in secondary analysis, zinc supplementation produced a greater reduction in glucose concentrations in those with chronic disease as compared to healthy participants. The dietary management of DM encourages the consumption of food sources that provide dietary fibre and PA, the latter contributing to an increased risk of zinc deficiency. The significant reduction in glucose concentrations suggests that zinc supplementation is a strategy to improve zinc status and contributes to 
the management of hyperglycaemia in individuals with chronic metabolic disease.

Insulin resistance and dyslipidaemia are linked to zinc metabolism potentially via zinc's role in signaling pathways and other metabolic processes [2]. Zinc homeostasis is regulated primarily by the activities of zinc transporter families, which are responsible for cellular zinc export (ZnT; SLC30) or influx (Zip; SLC39) [2]. We determined the gene expression of zinc transporters in peripheral blood mononuclear cells (PBMC) of women with DM [13]. Compared to the healthy group, the mRNA ratio of $Z n T 1$ (cellular zinc export) to Zip1 (cellular zinc import) was lower in participants with DM, which may indicate perturbed zinc homeostasis in the disorder, and potentially an increase in zinc requirements. In addition we investigated the relationship between measures of glycaemic control and expression of selected zinc transporters in a 12-week zinc supplementation trial involving patients with DM [15]. We found that fold change of Zip1O and ZnT6 predicted the change in serum glucose concentrations and insulin resistance, respectively. Taken together with reports of increased urinary zinc losses and lower concentrations of serum zinc in DM, these findings again suggest that it is beneficial to enhance the status of zinc in DM, by increasing zinc intake and/or decreasing factors that interfere with zinc bioavailability, namely PA.

\section{Zinc and inflammation}

As part of the pathology of disease, DM exhibits impaired immune function that ultimately contributes to decreased functional $\beta$-cell mass. Systemic pro-inflammatory markers, such as interleukins (IL)-1 $\beta$, and IL-6, often are linked to the progression of DM and nutrients such as zinc have been suggested to attenuate chronic inflammation associated with DM [16]. As with glycaemia, the mechanisms by which zinc modulates inflammation may be related to the role of zinc in cellular signaling pathways [16]. In a recent study in post-menopausal women with DM, we investigated the effect of zinc supplementation on inflammatory markers $[17,18]$. We identified a number of relationships among systemic inflammatory markers and gene expression of zinc transporters and cytokines. Specifically, systemic concentrations of IL-6 [17] and gene expression of IL-1 $\beta$ [18] were related to zinc transporter gene expression. These findings are suggestive of an attenuated immune response in DM after zinc supplementation through increased efficiency in signaling pathways. Inversely, the potentiation of cellular signals may be affected undesirably by an imbalance in the diet between intakes of zinc and inhibitors of zinc absorption, such as the large quantities of PA associated with fibre. Figure 1 shows the potential impact of PA and suboptimal zinc status on the management of chronic diseases.

\section{Conclusion}

The intake of foods that contain dietary fibre can influence favourably some but not all risk factors of chronic disease. Foods that are commonly included in therapeutic diets include legumes, wholegrain cereals, nuts and seeds - foods that are rich sources of PA, the most potent inhibitor of zinc absorption. Zinc deficiency is associated with a range of chronic conditions, including DM and cardiovascular disease. Recommendations to increase consumption of food sources of dietary fibre therefore need to be balanced against the need to maintain adequate zinc status. Dietary practices that increase zinc bioavailability, the consumption of foods fortified with zinc, or low dose zinc supplementation are strategies that should be considered for improving zinc status [10]. In addition, new strategies are required that will allow the degradation of PA without compromising the benefits of dietary fibre.

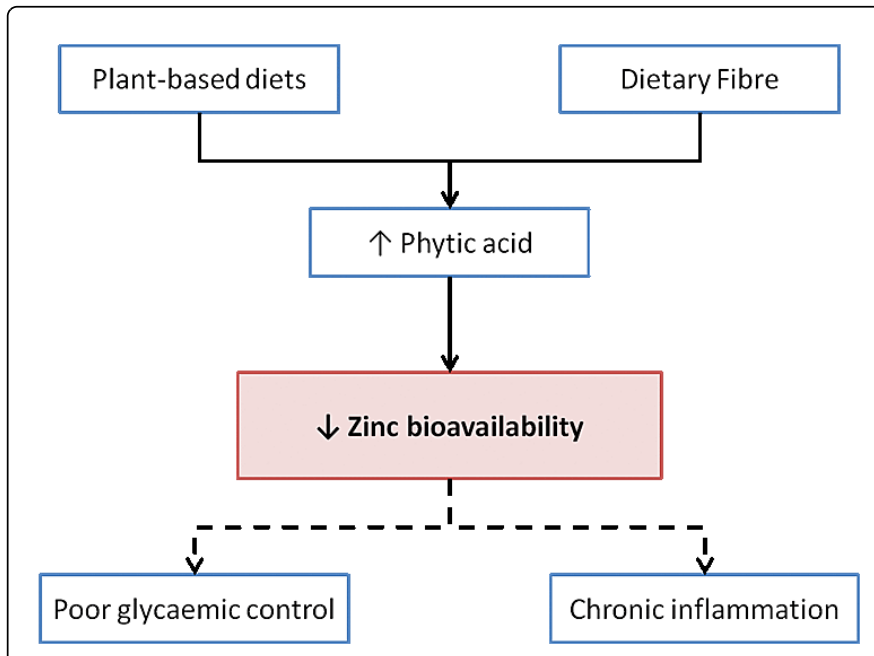

Figure 1. Phytic acid and zinc bioavailability in the management of chronic diseases. Plant-based diets and advice to consume more dietary fibre can increase the intake of phytic acid thereby reducing zinc bioavailability. Suboptimal zinc status negatively impacts glycaemic control and inflammation.

\section{References}

1. Samman S (2007) Zinc. Nutr Diet 64:S131-S134.

2. Foster M, Samman S (2010) Zinc and redox signaling: perturbations associated with cardiovascular disease and diabetes mellitus. Antioxid Redox Signal 13:1549-1573.

3. Sandström B (1997) Bioavailability of zinc. Eur J Clin Nutr 51:S17-S19.

4. World Health Organization (1996) Trace Elements in Human Nutrition and Health. Geneva: World Health Organization.

5. Oberleas D, Harland BF (1981) Phytate content of foods: effect on dietary zinc bioavailability. J Am Diet Assoc 79:433-436.

6. Anderson JW, Randles KM, Kendall CWC, Jenkins DJA (2004) Carbohydrate and fiber recommendations for individuals with diabetes: a quantitative assessment and meta-analysis of the evidence. J Am Coll Nutr 23:5-17.

7. Marinangeli CPF, Jones PJH (2012) Pulse grain consumption and obesity: effects on energy expenditure, substrate oxidation, body composition, fat deposition and satiety. Br J Nutr 108 Suppl: S46-51.

8. Foster M, Chu A, Petocz P, Samman S (2013) Effect of vegetarian diets on zinc status: a systematic review and meta-analysis of studies in humans. J Sci Food Agric 93:2362-2371.

9. Foster M, Samman S (2015) Vegetarian diets across the lifecycle: Impact on zinc intake and status. Adv Food Nutr Res. doi:10.1016/bs.afnr. 2014.11.003.

10. Samman S (2011) Challenges and opportunities in the assessment of zinc status. Nutr Diet 68:95-96.

11. Lonnerdal B, Mendoza C, Brown KH, Rutger JN, Raboy V (2011) Zinc absorption from low phytic acid genotypes of maize (Zea mays L.), Barley (Hordeum vulgare L.), and Rice (Oryza sativa L.) assessed in a suckling rat pup model. J Agric Food Chem 59:4755-4762.

12. Troesch B, van Stuijvenberg ME, Smuts CM, Kruger HS, Biebinger R, et al. (2011) A micronutrient powder with low doses of highly absorbable iron and zinc reduces iron and zinc deficiency and improves weight-for-age Zscores in South African children. J Nutr 141:237-242. 
Citation: Samir Samman* Anna Chu and Meika Foster (2015) Zinc and Dietary Fibre: Competing Nutrients in the Management of Chronic Diseases. Vitam Miner 4: e134. doi:10.4172/2376-1318.1000e134

Page 3 of 3

13. Foster M, Karra M, Picone T, Chu A, Hancock DP, et al. (2012) Dietary fiber intake increases the risk of zinc deficiency in healthy and diabetic women. Biol Trace Elem Res 149:135-142.

14. Capdor J, Foster M, Petocz P, Samman S (2013) Zinc and glycemic control: a meta-analysis of randomised placebo controlled supplementation trials in humans. J Trace Elem Med Biol 27:137-142.

15. Foster M, Chu A, Petocz P, Samman S (2014) Zinc transporter gene expression and glycemic control in post-menopausal women with type 2 diabetes mellitus. J Trace Elem Med Biol 28:448-452.
16. Foster M, Samman S (2012) Zinc and regulation of inflammatory cytokines: implications for cardiometabolic disease. Nutr 4:676-694.

17. Foster M, Petocz P, Samman S (2013) Inflammation markers predict zinc transporter gene expression in women with type 2 diabetes mellitus. J Nutr Biochem 24:1655-1661.

18. Chu A, Foster M, Hancock D, Bell-Anderson K, Petocz P, Samman S (2015) TNF- $\alpha$ gene expression is increased following zinc supplementation in type 2 diabetes mellitus. Genes Nutr 10:440. 